\title{
On the simplicity of induced modules for reductive Lie algebras. II
}

\author{
Chaowen Zhang \\ Department of Mathematics, \\ China University of Mining and Technology, \\ Xuzhou, 221116, Jiang Su, P. R. China
}

\begin{abstract}
Keywords and phrases: reductive Lie algebras, parabolic subalgebras, induced modules, simple modules.

Mathematics Subject Classification 2010: 17B10; 17B45; 17B50
\end{abstract}

\section{Introduction}

Let $G$ be a reductive algebraic group defined over an algebraically closed field $\mathbf{F}$ of positive characteristic $p$, and let $\mathfrak{g}$ be the Lie algebra of $G$. In [1, 5.1], Friedlander and Parshall asked to find necessary and sufficient conditions for the simplicity of a $\mathfrak{g}$-module with $p$-character $\chi \in \mathfrak{g}^{*}$ that is induced from a simple module for a parabolic subalgebra of $\mathfrak{g}$. This question has been answered (by V. Kac) when $\mathfrak{g}$ is of type $A_{2}$ (see [1, Example 3.6] and [6]), and also when $\mathfrak{g}$ is of type $A_{3}$ (see [7]). When $\mathfrak{g}$ is of type $A_{n}, B_{n}, C_{n}$ or $D_{n}$ and when $\chi$ is of standard Levi form, a sufficient condition is given in [8] for the simplicity of above-mentioned $\mathfrak{g}$-modules. Under certain assumption of $\chi$, a necessary and sufficient condition for the simplicity of these $\mathfrak{g}$-modules is given in [10]. In this paper we give a sufficient condition for the simplicity of these $\mathfrak{g}$-modules with a general assumption of $\chi$.

Following [4, 6.3] we make the following hypotheses:

(H1) The derived group $D G$ of $G$ is simply connected;

(H2) The prime $p$ is good for $\mathfrak{g}$;

(H3) There exists a $G$-invariant non-degenerate bilinear form on $\mathfrak{g}$.

Let $T$ be a maximal torus of $G$, let $\mathfrak{h}=\operatorname{Lie}(T)$, and let $\Phi$ be the root system of $G$. Let $\Pi=\left\{\alpha_{1}, \ldots, \alpha_{l}\right\}$ be a base of $\Phi$ and let $\Phi^{+}$be the set of positive roots relative to $\Pi$. For each $\alpha \in \Phi^{+}$let $\mathfrak{g}_{\alpha}$ denote the corresponding root space of $\mathfrak{g}$. 
According to [4, 6.1] we have $\mathfrak{g}=\mathfrak{n}^{-}+\mathfrak{h}+\mathfrak{n}^{+}$, where

$$
\mathfrak{n}^{+}=\sum_{\alpha \in \Phi^{+}} \mathfrak{g}_{\alpha}, \quad \mathfrak{n}^{-}=\sum_{\alpha \in \Phi^{+}} \mathfrak{g}_{-\alpha} .
$$

Fix a proper subset $I$ of $\Pi$ and put $\Phi_{I}=\mathbb{Z} I \cap \Phi$ and $\Phi_{I}^{+}=\Phi_{I} \cap \Phi^{+}$. Define $\tilde{\mathfrak{g}}_{I}=\mathfrak{h}+\sum_{\alpha \in \Phi_{I}} \mathfrak{g}_{\alpha}$, as well as

$$
\mathfrak{u}=\sum_{\alpha \in \Phi^{+} \backslash \Phi_{I}^{+}} \mathfrak{g}_{\alpha}, \quad \mathfrak{u}^{\prime}=\sum_{\alpha \in \Phi^{+} \backslash \Phi_{I}^{+}} \mathfrak{g}_{-\alpha} .
$$

Then $\mathfrak{p}_{I}=\tilde{\mathfrak{g}}_{I}+\mathfrak{u}$ is a parabolic subalgebras of $\mathfrak{g}$ with Levi factor $\tilde{\mathfrak{g}}_{I}$ [4, 10.6]. Throughout the paper we assume that $\chi\left(\mathfrak{n}^{+}\right)=0$. This is done without loss of generality due to [4, Lemma 6.6].

For any restricted Lie subalgebra $L$ of $\mathfrak{g}$, we denote by $u_{\chi}(L)$ the $\chi$-reduced enveloping algebra of $L$, where we continue to use $\chi$ for the restriction of $\chi$ to $L$ ([9, 5.3]). If $\chi=0$, then $u_{\chi}(L)$ is referred to as the restricted enveloping algebra of $L$, and denoted more simply by $u(L)$. Let $L_{I}^{\chi}(\lambda)$ be a simple $u_{\chi}\left(\mathfrak{p}_{I}\right)$-module generated by a maximal vector $v_{\lambda}$ of weight $\lambda \in \mathfrak{h}^{*}$. Define the induced $u_{\chi}(\mathfrak{g})$-module

$$
Z_{I}^{\chi}(\lambda)=u_{\chi}(\mathfrak{g}) \otimes_{u_{\chi}\left(\mathfrak{p}_{I}\right)} L_{I}^{\chi}(\lambda)
$$

The main result of the present paper is Theorem 2.10, which gives a sufficient condition for $Z_{I}^{\chi}(\lambda)$ to be simple; we show that $Z_{I}^{\chi}(\lambda)$ is simple if $\lambda$ is not a zero of a certain polynomial $R_{\mathfrak{g}}^{I}(\lambda)$. Then we give an application of this conclusion.

\section{Simplicity criterion}

In this section, we keep the assumptions as in the introduction. Let

$$
\left\{e_{\alpha}, h_{\beta} \mid \alpha \in \Phi, \beta \in \Pi\right\}
$$

be a Chevalley basis for $\mathfrak{g}^{\prime}=\operatorname{Lie}(D G)$ such that

$$
\left[e_{\alpha}, e_{\beta}\right]= \pm(r+1) e_{\alpha+\beta}, \quad \text { if } \quad \alpha, \beta, \alpha+\beta \in \Phi^{+},
$$

where $r$ is the greatest integer for which $\beta-r \alpha \in \Phi$ (see [3, Theorem 25.2]). As pointed out in [10, Section 3], our assumption on $p$ ensures that $(r+1) \neq 0$.

For $\alpha \in \Phi^{+}$put $f_{\alpha}=-e_{\alpha}$. Then we have $\mathfrak{g}_{\alpha}=\mathbf{F} e_{\alpha}$ and $\mathfrak{g}_{-\alpha}=\mathbf{F} f_{\alpha}$ for every $\alpha \in \Phi^{+}$.

Remark: Let $\alpha, \beta \in \Phi^{+}$such that $\alpha+\beta \in \Phi^{+}\left(\operatorname{resp} . \beta-\alpha \in \Phi^{+}\right)$. Then we have

$$
\left[e_{\alpha}, e_{\beta}\right]=c e_{\alpha+\beta}, \quad\left[f_{\alpha}, f_{\beta}\right]=-c f_{\alpha+\beta}\left(\operatorname{resp} .\left[e_{\alpha}, f_{\beta}\right]=c f_{\beta-\alpha}\right)
$$


for some $c \in \mathbf{F} \backslash 0$. For brevity, we omit the scalar $c$. This does not affect any of the proofs in this section.

Recall from the introduction the notation $\mathfrak{p}_{I}, \mathfrak{u}, \mathfrak{u}^{\prime}, \tilde{\mathfrak{g}}_{I}$, and $L_{I}^{\chi}(\lambda)$. Using (H3), we can show that $\mathfrak{u}$ is the nilradical of the parabolic subalgebra $\mathfrak{p}_{I}$. By [9, Corollary 1.3.8], $L_{I}^{\chi}(\lambda)$ is annihilated by $\mathfrak{u}$, and hence is a simple $u_{\chi}\left(\tilde{\mathfrak{g}}_{I}\right)$-module. The simple $u_{\chi}\left(\mathfrak{p}_{I}\right)$-module $L_{I}^{\chi}(\lambda)$ is generated by any of its maximal vectors, which may not be unique under our assumption.

Recall from the introduction the induced $u_{\chi}(\mathfrak{g})$-module $Z_{I}^{\chi}(\lambda)$. By the PBW theorem for the $\chi$-reduced enveloping algebra $u_{\chi}(\mathfrak{g})([9$, Theorem 5.3.1]), we have

$$
Z_{I}^{\chi}(\lambda) \cong u_{\chi}\left(\mathfrak{u}^{\prime}\right) \otimes_{\mathbf{F}} L_{I}^{\chi}(\lambda)
$$

as $u_{\chi}\left(\mathfrak{u}^{\prime}\right)$-modules.

Let $\Phi^{+} \backslash \Phi_{I}^{+}=\left\{\beta_{1}, \beta_{2}, \ldots, \beta_{k}\right\}$, and let $v_{1}, \ldots, v_{n}$ be a basis of $L_{I}^{\chi}(\lambda)$. Then $Z_{I}^{\chi}(\lambda)$ has a basis

$$
f_{\beta_{1}}^{l_{1}} f_{\beta_{2}}^{l_{2}} \cdots f_{\beta_{k}}^{l_{k}} \otimes v_{j}, \quad 0 \leq l_{i} \leq p-1, i=1, \ldots, k, j=1, \ldots, n .
$$

Let $\mathfrak{S}$ be a subset of $\Phi^{+}$. We say that $\mathfrak{S}$ is a closed subset if $\alpha+\beta \in \mathfrak{S}$ for any $\alpha, \beta \in \mathfrak{S}$ such that $\alpha+\beta \in \Phi^{+}$. Therefore, $\Phi^{+} \backslash \Phi_{I}^{+}$is a closed subset of $\Phi^{+}$. We see that $\mathfrak{S}$ is a closed subset of $\Phi^{+}$if and only if $\mathfrak{s}=: \sum_{\alpha \in \mathfrak{S}} \mathfrak{g}_{\alpha}$ is a Lie subalgebra of $\mathfrak{g}$; it is clear that $\mathfrak{s}$ is restricted.

Let $\mathfrak{S}$ be a closed subset of $\Phi^{+}$. Applying almost verbatim Humphreys's argument in the proof of [2, Lemma 1.4], we get the following result.

Lemma 2.1. Let $\left(\alpha_{1}, \ldots, \alpha_{m}\right)$ be any ordering of $\mathfrak{S}$. If $h t\left(\alpha_{k}\right)=h$, assume that all exponents $i_{j}$ in $e_{\alpha_{1}}^{i_{1}} \cdots e_{\alpha_{m}}^{i_{m}} \in u(\mathfrak{s})$ for which $h t\left(\alpha_{j}\right) \geq h$ are equal to $p-1$. Then, if $e_{\alpha_{k}}$ is inserted anywhere into this expression, the result is 0 .

Corollary 2.2. Let $\Phi^{+} \backslash \Phi_{I}^{+}=\left\{\beta_{1}, \ldots, \beta_{k}\right\}$. For $e_{\beta_{1}}^{p-1} \cdots e_{\beta_{k}}^{p-1} \in u(\mathfrak{u})$, we have in $u_{\chi}(\mathfrak{g})$ that

$$
\text { (1) }\left[e_{\alpha}, e_{\beta_{1}}^{p-1} \cdots e_{\beta_{k}}^{p-1}\right]=0,(2)\left[f_{\alpha}, e_{\beta_{1}}^{p-1} \cdots e_{\beta_{k}}^{p-1}\right]=0
$$

for every $\alpha \in \Phi_{I}^{+}$.

Proof. (1) is immediate from Lemma 2.1.

(2) is given by [10, Lemma 3.5].

In the following we assume $\beta_{1}, \ldots, \beta_{k}$ in $\Phi^{+} \backslash \Phi_{I}^{+}$is in the order of ascending heights. By [9, Theorem 3.3.1], $u_{\chi}\left(\mathfrak{u}^{\prime}\right)$ has a basis

$$
f_{\beta_{1}}^{l_{1}} \cdots f_{\beta_{k}}^{l_{k}}, \quad 0 \leq l_{i} \leq p-1 .
$$

Lemma 2.3. Let $1 \leq s \leq k$, and let $f_{\beta_{s}}^{l_{s}} \cdots f_{\beta_{k}}^{l_{k}} \in u_{\chi}\left(\mathfrak{u}^{\prime}\right), 0 \leq l_{s}, \ldots, l_{k} \leq p-1$. For any $j \geq s$, the product $f_{\beta_{j}} f_{\beta_{s}}^{l_{s}} \cdots f_{\beta_{k}}^{l_{k}}$ equals a linear combination of elements $f_{\beta_{s}}^{i_{s}} \cdots f_{\beta_{k}}^{i_{k}}$ such that $0 \leq i_{s}, \ldots, i_{k} \leq p-1$. 
Proof. For $\beta_{i}, \beta_{j}$ in $\Phi^{+} \backslash \Phi_{I}^{+}$, if $\beta_{i}+\beta_{j}$ is also a root, then its height is greater than those of both $\beta_{i}$ and $\beta_{j}$. This implies that

$$
\mathfrak{u}_{s}^{\prime}=:\left\langle f_{\beta_{s}}, \ldots, f_{\beta_{k}}\right\rangle
$$

is a (restricted) Lie subalgebra of $\mathfrak{u}^{\prime}$. Then [9, Theorem 3.3.1] says that $u_{\chi}\left(\mathfrak{u}_{s}^{\prime}\right)$, viewed as a subalgebra of $u_{\chi}\left(\mathfrak{u}^{\prime}\right)$, has a basis

$$
f_{\beta_{s}}^{l_{s}} \cdots f_{\beta_{k}}^{l_{k}}, \quad 0 \leq l_{s}, \ldots, l_{k} \leq p-1
$$

Then the lemma follows.

Lemma 2.4. For $\alpha \in I$, the element $\left[e_{\alpha}, f_{\beta_{1}}^{p-1} \cdots f_{\beta_{k}}^{p-1}\right] \in u_{\chi}(\mathfrak{g})$ equals a linear combination of basis vectors

$$
f_{\beta_{1}}^{l_{1}} \cdots f_{\beta_{k}}^{l_{k}}, \quad 0 \leq l_{i} \leq p-1
$$

of $u_{\chi}\left(\mathfrak{u}^{\prime}\right)$ such that $l_{i}<p-1$ for some $i=1, \ldots, k$.

Proof. If $\left[e_{\alpha}, f_{\beta_{i}}\right] \neq 0$ for some $i$, then since $\alpha$ is a simple root, we have

$$
\left[e_{\alpha}, f_{\beta_{i}}\right]=f_{\beta_{i}-\alpha}
$$

with $\beta_{i}-\alpha=\beta_{j}$ for some $j=1, \ldots, k$. Clearly we have $\operatorname{ht}\left(\beta_{i}-\alpha\right)<\operatorname{ht} \beta_{i}$. For

$$
\left[e_{\alpha}, f_{\beta_{1}}^{p-1} \cdots f_{\beta_{k}}^{p-1}\right]=\sum_{i,\left[e_{\alpha}, f_{\beta_{i}}\right] \neq 0} f_{\beta_{1}}^{p-1} \cdots f_{\beta_{i}-\alpha}^{p-1} \cdots\left(\sum_{s=0}^{p-2} f_{\beta_{i}}^{s} f_{\beta_{i}-\alpha} f_{\beta_{i}}^{p-2-s}\right) \cdots f_{\beta_{k}}^{p-1}
$$

each summand equals

$$
\begin{gathered}
(p-1) f_{\beta_{1}}^{p-1} \cdots f_{\beta_{i}-\alpha}^{p} \cdots f_{\beta_{i}}^{p-2} \cdots f_{\beta_{k}}^{l_{k}} \\
+\sum_{s=0}^{p-2} f_{\beta_{1}}^{p-1} \cdots f_{\beta_{i}-\alpha}^{p-1}\left[\cdots f_{\beta_{i}}^{s}, f_{\beta_{i}-\alpha}\right] f_{\beta_{i}}^{p-2-s} \cdots f_{\beta_{k}}^{l_{k}} .
\end{gathered}
$$

Since $f_{\beta_{i}-\alpha}^{p}=\chi\left(f_{\beta_{i}-\alpha}\right)^{p}$, the first term above is of the desired form. For each term in the summation above, if there is $\beta_{t}$ between $\beta_{i}-\alpha$ and $\beta_{i}$ (including $\beta_{i}$ ) such that

$$
f_{\beta}=:\left[f_{\beta_{t}}, f_{\beta_{i}-\alpha}\right] \neq 0,
$$

so that ht $\beta>$ ht $\beta_{t}$, then by Lemma 2.3 this term equals a linear combination of elements $f_{\beta_{1}}^{l_{1}} \cdots f_{\beta_{t}}^{l_{t}} \cdots f_{\beta_{k}}^{l_{k}}, 0 \leq l_{i} \leq p-1$, with especially $l_{t}<p-1$. Thus, the lemma holds.

Lemma 2.5. For $\alpha \in I,\left[f_{\alpha}, f_{\beta_{1}}^{p-1} \cdots f_{\beta_{k}}^{p-1}\right] \in u_{\chi}(\mathfrak{g})$ equals a linear combination of of basis vectors

$$
f_{\beta_{1}}^{l_{1}} \cdots f_{\beta_{k}}^{l_{k}}, \quad 0 \leq l_{i} \leq p-1
$$

of $u_{\chi}\left(\mathfrak{u}^{\prime}\right)$ such that $l_{i}<p-1$ for some $i=1, \ldots, k$. 
Proof. If $\left[f_{\alpha}, f_{\beta_{i}}\right] \neq 0$ for some $i$, so that $\left[f_{\alpha}, f_{\beta_{i}}\right]=f_{\beta_{j}}$ for some $j>i$ since ht $\beta_{j}>$ ht $\beta_{i}$. Applying Lemma 2.3 we see that

$$
f_{\beta_{1}}^{p-1} \cdots\left[f_{\alpha}, f_{\beta_{i}}^{p-1}\right] \cdots f_{\beta_{k}}^{p-1}
$$

is a linear combination of $f_{\beta_{1}}^{l_{1}} \cdots f_{\beta_{i}}^{l_{i}} \cdots f_{\beta_{k}}^{l_{k}}$ with $0 \leq l_{1}, \ldots, l_{i}, \ldots, l_{k} \leq p-1$, and in particular, $l_{i}=p-2<p-1$. Then the lemma follows.

By a similar proof as that for [10, Lemma 3.6], we obtain the following lemma.

Lemma 2.6. There is a uniquely determined scalar $R_{\mathfrak{g}}^{I}(\lambda) \in \mathbf{F}$ such that

$$
e_{\beta_{1}}^{p-1} \cdots e_{\beta_{k}}^{p-1} f_{\beta_{1}}^{p-1} \cdots f_{\beta_{k}}^{p-1} \otimes v_{l}=R_{\mathfrak{g}}^{I}(l) \otimes v_{\lambda}
$$

in $Z_{I}^{\chi}(\lambda)$.

Let $\chi \in \mathfrak{g}^{*}$ as given earlier. Then $\chi$ can be written as $\chi=\chi_{s}+\chi_{n}$, with $\chi_{s}\left(\mathfrak{n}^{+}+\mathfrak{n}^{-}\right)=0$ and $\chi_{n}\left(\mathfrak{h}+\mathfrak{n}^{+}\right)=0$. For each simple $u_{\chi_{s}}\left(\mathfrak{p}_{I}\right)$-module $L_{I}^{\chi_{s}}(\lambda)$ $\left(\lambda \in \mathfrak{h}^{*}\right)$, define the induced module

$$
Z_{I}^{\chi_{s}}(\lambda)=u_{\chi_{s}}(\mathfrak{g}) \otimes_{u_{\chi_{s}}\left(\mathfrak{p}_{I}\right)} L_{I}^{\chi_{s}}(\lambda) .
$$

Let $v_{l} \in L_{I}^{\chi_{s}}(\lambda)$ be a maximal vector of weight $\lambda$. In a similar way as above we define the scalar $R_{\mathfrak{g}}^{I}(\lambda)_{s}$ by

$$
e_{\beta_{1}}^{p-1} \cdots e_{\beta_{k}}^{p-1} f_{\beta_{1}}^{p-1} \cdots f_{\beta_{k}}^{p-1} \otimes v_{\lambda}=R_{\mathfrak{g}}^{I}(\lambda)_{s} \otimes v_{\lambda} .
$$

We have $R_{\mathfrak{g}}^{I}(\lambda)_{s}=R_{\mathfrak{g}}^{I}(\lambda)$ for any $\lambda \in \mathfrak{h}^{*}$ by a similar proof as that for [10, Lemma 4.1]. Therefore, in calculating $R_{\mathfrak{g}}^{I}(\lambda)$, we may assume $\chi=\chi_{s}$. By [10, Lemma 4.1], there is nonzero $c \in \mathbf{F}$ (independent of $l$ ) such that

$$
R_{\mathfrak{g}}^{I}(\lambda)=c \Pi_{i=1}^{k}\left[(\lambda+\rho)\left(h_{\beta_{i}}\right)^{p-1}-1\right], \quad l \in \mathfrak{h}^{*} .
$$

We now put the basis elements of $\mathfrak{n}^{+}$in the order

$$
e_{\alpha_{1}}, \ldots, e_{\alpha_{s}}, e_{\beta_{1}}, \ldots, e_{\beta_{k}}
$$

for which $\left\{\alpha_{1}, \ldots, \alpha_{s}\right\}=\Phi_{I}^{+}$.

Let $U(\mathfrak{g})$ (resp. $\left.U(\mathfrak{h}) ; U\left(\mathfrak{n}^{+}\right)\right)$be the universal enveloping algebra of $\mathfrak{g}\left(\mathfrak{h} ; \mathfrak{n}^{+}\right)$. Then $U(\mathfrak{g})$ is naturally a $T$-module with the adjoint action. Let us denote the $T$-weight of a weight vector $u \in U(\mathfrak{g})$ by $\operatorname{wt}(u)$.

Assume $l=\left(l_{1}, \ldots, l_{k}\right) \in \mathbb{N}^{k}$ with $l_{i} \leq p-1$. Then we have in $U(\mathfrak{g})$

$$
x=e_{\beta_{1}}^{p-1} \cdots e_{\beta_{k}}^{p-1} f_{\beta_{1}}^{l_{1}} \cdots f_{\beta_{k}}^{l_{k}}=\sum_{i} u_{i}^{-} u_{i}^{0} u_{i}^{+}, \quad u_{i}^{-} \in U\left(\mathfrak{n}^{-}\right), u_{i}^{0} \in U(\mathfrak{h}), u_{i}^{+} \in U\left(\mathfrak{n}^{+}\right) .
$$

Under the $T$-action, the weight of $x$ is

$$
\operatorname{wt}(x)=\operatorname{wt}\left(u_{i}^{+}\right)+\operatorname{wt}\left(u_{i}^{-}\right)=\sum_{i, l_{i}<p-1}\left(p-1-l_{i}\right) \beta_{i} .
$$


Lemma 2.7. Keep the assumptions above. If one of the exponents $l_{1}, \ldots, l_{k}$ is strictly less than $p-1$, then for each $i$ we have

$$
u_{i}^{+}=e_{\alpha_{1}}^{j_{1}} \cdots e_{\alpha_{s}}^{j_{s}} e_{\beta_{1}}^{t_{1}} \cdots e_{\beta_{k}}^{t_{k}}, \quad j_{1}, \ldots, j_{s}, t_{1}, \ldots, t_{k} \in \mathbb{N}
$$

such that $t_{q}>0$ for some $q=1, \ldots, k$.

Proof. Suppose otherwise that $t_{q}=0$ for all $q$. Then we have

$$
\mathrm{wt}\left(u_{i}^{+}\right)=\sum_{i=1}^{s} j_{i} \alpha_{i} \in \mathbb{N} I .
$$

Note that

$$
\operatorname{wt}\left(u_{i}^{+}\right)=-\operatorname{wt}\left(u_{i}^{-}\right)+\sum_{i, l_{i}<p-1}\left(p-1-l_{i}\right) \beta_{i} .
$$

Expressed as linear combinations of simple roots $\Pi,-$ wt $\left(u_{i}^{-}\right)$has all coefficients nonnegative, but

$$
\sum_{i, l_{i}<p-1}\left(p-1-l_{i}\right) \beta_{i}
$$

has at least a positive coefficient for some simple root in $\Pi \backslash I$, a contradiction.

Lemma 2.8. Keep the assumptions above. If one of the exponents $l_{1}, \ldots, l_{k}$ is strictly less than $p-1$, then we have in $Z_{I}^{\chi}(l)$ that

$$
e_{\beta_{1}}^{p-1} \ldots e_{\beta_{k}}^{p-1} f_{\beta_{1}}^{l_{1}} \cdots f_{\beta_{k}}^{l_{k}} \otimes v=0 \quad \text { for any } v \in L_{I}^{\chi}(l) .
$$

Proof. By our assumptions, we have in $U(\mathfrak{g})$ that

$$
e_{\beta_{1}}^{p-1} \ldots e_{\beta_{k}}^{p-1} f_{\beta_{1}}^{l_{1}} \cdots f_{\beta_{k}}^{l_{k}}=\sum u_{i}^{-} u_{i}^{0} u_{i}^{+}, u_{i}^{-} \in U\left(\mathfrak{n}^{-}\right), u_{i}^{0} \in U(\mathfrak{h}), u_{i}^{+} \in U\left(\mathfrak{n}^{+}\right),
$$

where by Lemma 2.7 each $u_{i}^{+}$is of the form

$$
e_{\alpha_{1}}^{j_{1}} \cdots e_{\alpha_{s}}^{j_{s}} e_{\beta_{1}}^{t_{1}} \cdots e_{\beta_{k}}^{t_{k}}
$$

with $t_{q}>0$ for some $q$. Then we have in $u_{\chi}(\mathfrak{g})$ that

$$
e_{\beta_{1}}^{p-1} \cdots e_{\beta_{k}}^{p-1} f_{\beta_{1}}^{l_{1}} \cdots f_{\beta_{k}}^{l_{k}}=\sum \bar{u}_{i}^{-} \bar{u}_{i}^{0} \bar{u}_{i}^{+}, \quad \bar{u}_{i}^{-} \in u_{\chi}\left(\mathfrak{n}^{-}\right), \bar{u}_{i}^{0} \in u_{\chi}(\mathfrak{h}), u_{i}^{+} \in u\left(\mathfrak{n}^{+}\right) .
$$

For each $u_{i}^{+}$of the above form, we have $\bar{u}_{i}^{+}=0$ if one of the exponents

$$
j_{1}, \ldots, j_{s}, t_{1}, \ldots, t_{k}
$$

is greater than or equal to $p$. If all these exponents are less than $p$, then $\bar{u}_{i}^{+}$is a basis vector of $u\left(\mathfrak{n}^{+}\right)$of the same form as $u_{i}^{+}$, and hence $\bar{u}_{i}^{+} \otimes v=0$ since one of the $t_{q}$ is positive. Therefore we have $\bar{u}_{i}^{+} \otimes v=0$ for all $i$, so the lemma follows. 
Lemma 2.9. Keep the assumptions above. Let $v \in L_{I}^{\chi}(l)$. For any $\alpha \in I$, we have in $Z_{I}^{\chi}(l)$ that

(1) $e_{\alpha} e_{\beta_{1}}^{p-1} \cdots e_{\beta_{k}}^{p-1} f_{\beta_{1}}^{p-1} \cdots f_{\beta_{k}}^{p-1} \otimes v=e_{\beta_{1}}^{p-1} \cdots e_{\beta_{k}}^{p-1} f_{\beta_{1}}^{p-1} \cdots f_{\beta_{k}}^{p-1} \otimes e_{\alpha} v$,

(2) $\quad f_{\alpha} e_{\beta_{1}}^{p-1} \cdots e_{\beta_{k}}^{p-1} f_{\beta_{1}}^{p-1} \cdots f_{\beta_{k}}^{p-1} \otimes v=e_{\beta_{1}}^{p-1} \cdots e_{\beta_{k}}^{p-1} f_{\beta_{1}}^{p-1} \cdots f_{\beta_{k}}^{p-1} \otimes f_{\alpha} v$.

Proof. (1) By Corollary 2.2, the left side equals

$$
\begin{gathered}
e_{\beta_{1}}^{p-1} \cdots e_{\beta_{k}}^{p-1} e_{\alpha} f_{\beta_{1}}^{p-1} \cdots f_{\beta_{k}}^{p-1} \otimes v=e_{\beta_{1}}^{p-1} \cdots e_{\beta_{k}}^{p-1} f_{\beta_{1}}^{p-1} \cdots f_{\beta_{k}}^{p-1} \otimes e_{\alpha} v \\
+e_{\beta_{1}}^{p-1} \cdots e_{\beta_{k}}^{p-1}\left[e_{\alpha}, f_{\beta_{1}}^{p-1} \cdots f_{\beta_{k}}^{p-1}\right] \otimes v \\
=e_{\beta_{1}}^{p-1} \cdots e_{\beta_{k}}^{p-1} f_{\beta_{1}}^{p-1} \cdots f_{\beta_{k}}^{p-1} \otimes e_{\alpha} v
\end{gathered}
$$

where the last equality follows from Lemma 2.4 and 2.8.

(2) follows from Corollary 2.2, Lemma 2.5 and 2.8.

Theorem 2.10. The $u_{\chi}(\mathfrak{g})$-module $Z_{I}^{\chi}(\lambda)$ is simple if $R_{\mathfrak{g}}^{I}(\lambda) \neq 0$.

Proof. Suppose $R_{\mathfrak{g}}^{I}(\lambda) \neq 0$. Let $N$ be a nonzero submodule of $Z_{I}^{\chi}(\lambda)$, and let $x \in N$ be a nonzero element, which we can write

$$
x=\sum_{l} c_{l} f_{\beta_{1}}^{l_{1}} \cdots f_{\beta_{k}}^{l_{k}} \otimes v_{l}
$$

where the sum is over all tuples $l=\left(l_{1}, \ldots, l_{k}\right)$ with $0 \leq l_{i} \leq p-1$ and where $c_{l} \in \mathbf{F}$ and $0 \neq v_{l} \in L_{I}^{\chi}(l)$.

First, applying $f_{\beta_{i}}$ 's with $\chi\left(f_{\beta_{i}}\right)=0$ in the order of descending heights, then applying $f_{\beta_{i}}$ 's with $\chi\left(f_{\beta_{i}}\right) \neq 0$ in the order of ascending heights, we get

$$
x^{\prime}=\sum c_{l} f_{\beta_{1}}^{l_{1}} \cdots f_{\beta_{k}}^{l_{k}} \otimes v_{l} \in N, \quad 0 \leq l_{i} \leq p-1,
$$

where for each $l=\left(l_{1}, \ldots, l_{k}\right)$ with $c_{l} \neq 0$, we have $l_{j}=p-1$ if $\chi\left(f_{\beta_{j}}\right)=0$, but we may have $l_{i}<p-1$ if $\chi\left(f_{\beta_{i}}\right) \neq 0$. In particular, we have $c_{\hat{l}} \neq 0$ for

$$
\hat{l}=(p-1, \ldots, p-1) .
$$

It follows that

$$
e_{\beta_{1}}^{p-1} \cdots e_{\beta_{k}}^{p-1} x^{\prime}=\sum_{l} c_{l} e_{\beta_{1}}^{p-1} \ldots e_{\beta_{k}}^{p-1} f_{\beta_{1}}^{l_{1}} \cdots f_{\beta_{k}}^{l_{k}} \otimes v_{l} \in N .
$$

If $l \neq \hat{l}$, that is, $l_{i}<p-1$ for some $\beta_{i}$, then we have by Lemma 2.8 that

$$
c_{l} e_{\beta_{1}}^{p-1} \ldots e_{\beta_{k}}^{p-1} f_{\beta_{1}}^{l_{1}} \cdots f_{\beta_{k}}^{l_{k}} \otimes v_{l}=0
$$


and hence

$$
e_{\beta_{1}}^{p-1} \cdots e_{\beta_{k}}^{p-1} x^{\prime}=c_{\hat{l}} e_{\beta_{1}}^{p-1} \cdots e_{\beta_{k}}^{p-1} f_{\beta_{1}}^{p-1} \cdots f_{\beta_{k}}^{p-1} \otimes v_{\hat{l}} \in N .
$$

Let $v_{l} \in L_{I}^{\chi}(l)$ be a maximal vector of weight $l$. Since $L_{I}^{\chi}(l)$ is simple as a $u_{\chi}\left(\tilde{\mathfrak{g}}_{I}\right)$ module, there is

$$
u=\sum u_{i}^{-} u_{i}^{0} u_{i}^{+} \in u_{\chi}\left(\tilde{\mathfrak{g}}_{I}\right)
$$

such that $u v_{\hat{l}}=v_{l}$. It is clear that

$$
\left[h, e_{\beta_{1}}^{p-1} \cdots e_{\beta_{k}}^{p-1} f_{\beta_{1}}^{p-1} \cdots f_{\beta_{k}}^{p-1}\right]=0
$$

for all $h \in \mathfrak{h}$. Then by Lemma 2.9 and 2.6 we have

$$
\begin{aligned}
u e_{\beta_{1}}^{p-1} \cdots e_{\beta_{k}}^{p-1} f_{\beta_{1}}^{p-1} \cdots f_{\beta_{k}}^{p-1} \otimes v_{\hat{l}} & =e_{\beta_{1}}^{p-1} \cdots e_{\beta_{k}}^{p-1} f_{\beta_{1}}^{p-1} \cdots f_{\beta_{k}}^{p-1} \otimes u v_{\hat{l}} \\
& =e_{\beta_{1}}^{p-1} \cdots e_{\beta_{k}}^{p-1} f_{\beta_{1}}^{p-1} \cdots f_{\beta_{k}}^{p-1} \otimes v_{l} \\
& =R_{\mathfrak{g}}^{I}(l) \otimes v_{l} \in N,
\end{aligned}
$$

implying that $1 \otimes v_{l} \in N$, and hence $N=Z_{I}^{\chi}(l)$. Thus, $Z_{I}^{\chi}(\lambda)$ is simple.

In [1, 5.1], Friedlander and Parshall also asked, for $\chi \in \mathfrak{g}^{*}$, which $u_{\chi}(\mathfrak{g})$-modules are induced from a restricted module for some proper restricted subalgebra of $\mathfrak{g}$. 8 , Theorem 1.2] partially answers this question. We now study this question as an application of the above theorem.

Keep the notation from the introduction. We assume that $\chi\left(\mathfrak{h}+\mathfrak{n}^{+}\right)=0$. Assume that there is a subset $I$ of $\Pi$ such that $\chi\left(\mathfrak{p}_{I}\right)=0$. Then $Z_{I}^{\chi}(\lambda)$ is induced from a restricted simple $\mathfrak{p}_{I}$-module.

For example, if $\chi$ is in the standard Levi form, i.e., there is a subset $J \subseteq \Pi$ such that $\chi\left(f_{\alpha}\right) \neq 0$ for all $\alpha \in J$, and $\chi\left(f_{\alpha}\right)=0$ for all $\alpha \in \Phi^{+} \backslash J$, we take $I=\Pi \backslash J$.

Recall the maximal torus $T$ of $G$ from the introduction. Let $X(T)$ be the character group of $T$. A weight $\tilde{\mu} \in X(T)$ is $p$-regular if its stabilizer in the affine Weyl group of $\mathfrak{g}$ is trivial. By [5, p.225], the differential of $\tilde{\mu}$ is an element in $\mu \in \mathfrak{h}^{*}$ satisfying $\mu\left(h^{[p]}\right)=\mu(h)^{p}$ for all $h \in \mathfrak{h}$.

Now let $\lambda \in \mathfrak{h}^{*}$ be the differential of a weight $\tilde{\lambda} \in X(T)$ such that $R_{\mathfrak{g}}^{I}(\lambda) \neq 0$. Using the notation from Section 2, we have

$$
\Phi^{+} \backslash \Phi_{I}^{+}=\left\{\beta_{1}, \ldots, \beta_{k}\right\} .
$$

Then $R_{\mathfrak{g}}^{I}(\lambda) \neq 0$ implies that $(\lambda+\rho)\left(h_{\beta_{i}}\right)=0$ for all $i$, so that the weight $\tilde{\lambda}$ is not $p$-regular. But Theorem 2.10 says that $Z_{I}^{\chi}(\lambda)$ is simple. Therefore, $Z_{I}^{\chi}(\lambda)$ is not included in those constructed in [8, Theorem 1.2] in the case that $\chi$ is in the standard Levi form. 


\section{REFERENCES}

[1] E. Friedlander and B. Parshall, Deformations of Lie algebra representations, Amer. J. Math. 112 (1990), 375-395.

[2] J. E. Humphreys, Modular representations of classical Lie algebras and semisimple groups, J. Algebra 19 (1971), 51-79.

[3] J. E. Humphreys, Introduction to Lie algebras and representation theory, Springer-Verlag, 1972.

[4] J. C. Jantzen, Representations of Lie algebras in prime characteristic, in: A. Borel (Ed.), Proceedings, Montreal, 1997, in: NATO ASI Series, V. C 514, Kluwer, Dordrecht (1998), 185-235.

[5] J. C. Jantzen, Subregular nilpotent representations of $\mathfrak{s l}_{n}$ and $\mathfrak{s o}_{2 n+1}$, Math. Proc. Camb. Phil. Soc. 23 (1999), 223-257.

[6] V. Kac, On irreducible representations of Lie algebras of classical type (Russian), Uspekhi Mat. Nauk 27 (1972), 237-238.

[7] Y. Li, B. Shu, and Y. Yao, A necessary and sufficient condition for irreducibility of parabolic baby Verma modules of $\mathfrak{s l}(4, k)$, J. Pure and Appl. Algebra 219 (2015), 760-766.

[8] Y. Li, B. Shu, and Y. Yao, A criterion for irreducibility of parabolic baby Verma modules of reductive Lie algebras, arXiv:1404.4945.

[9] H. Strade and R. Farnsteiner, Modular Lie algebras and their representations, Pure and Appl. Math. 116, Math. Marcel Dekker, 1988.

[10] C. Zhang, On the simplicity of induced modules for reductive Lie algebras, Bull. Belg. Math. Soc. Simon Stevin 23 (2016), 357-371. 